MAXIME HURÉ*

ARnaud PASSALACQUA**

\title{
Un changement de braquet dans l'action municipale des années 1970 ?
}

\author{
L'expérience des vélos en libre-service \\ de La Rochelle et la transformation \\ de l'action publique urbaine
}

D armi les évolutions récentes de la mobilité urbaine, les systèmes de vélos en libre-service frappent par la rapidité avec laquelle les villes européennes se sont dotées de tels équipements, ainsi que par le jeu d'acteurs très spécifique qui se trouve derrière eux, et par le large succès dont ils jouissent généralement ${ }^{1}$. Ce phénomène récent a éclipsé une expérience précédente, celle engagée à La Rochelle en 1976, qui avait pourtant posé les fondements des systèmes de vélos en libre-service contemporains. En effet, il marquait l'ébauche des discours environnementaux institutionnels en faveur du vélo. C'est aussi à ce moment qu'a émergé le jeu d'acteurs à l'œuvre derrière ces systèmes, croisant élus municipaux et entreprises. Mieux, cette première expérience permet d'interroger l'apport des dynamiques politiques des années 1970 à l'organisation de la mobilité en ville.

Elle pose effectivement la question des transformations de l'action publique urbaine en matière de mobilité. Dans les années 1970, l'action

\footnotetext{
* UMR Triangle, action, discours, pensée politique et économique, 5206 CNRS, Sciences po Lyon, ENS de Lyon.

** Université Paris Diderot - ICT EA 337.

1. Cet article s'appuie notamment sur les fonds des Archives municipales de La Rochelle (désormais AMLR). Il a bénéficié d'une aide de l'Agence nationale de la recherche portant la référence "ANR-09-SSOC-036», projet Resendem "Les grands réseaux techniques en démocratie: innovation, usages et groupes impliqués dans la longue durée, des années 1880 à nos jours ».
} 
des institutions municipales est perçue comme fortement dépendante des services de l'État. Les liens entre le ministère de l'Équipement et les pouvoirs urbains s'organisent alors selon une vision centrée sur l'État. Les relations entre les élus et l'administration entrent généralement dans le cadre de la "régulation croisée ${ }^{2}$ qui consiste en des arrangements entre techniciens de l'État et élus, dont certains sont les porteurs des projets étatiques. Cependant, l'État fait face à l'émergence de questions nouvelles issues du terrain local, parmi lesquelles celle du vélo en ville ${ }^{3}$.

L'expérience pionnière de vélos en libre-service que connaît La Rochelle à cette époque s'inscrit dans cette dynamique politique et sociale, héritière des mouvements sociaux urbains de Mai 1968, sous la forme du concept central mais ambivalent de "banalisation ". Porteuse d'une vision nouvelle de ce que doivent être une ville et l'organisation de ses déplacements, elle présente indéniablement un caractère novateur, bien perçu à l'époque.

Pour autant, la nature de cette innovation mérite réflexion. Est-elle technique et concrétisée par les vélos, stations et tarifs? Est-elle institutionnelle et digne d'intérêt $\mathrm{du}$ fait des formes adoptées pour piloter ce projet, de la création d'une commission extra-municipale à la mise en place d'un partenariat avec le Crédit agricole? Est-elle sociale à travers son appropriation par les Rochelais comme par les touristes? L'ambition est-elle de rénover un centre-ville et de le désengorger des voitures? Ou de promouvoir le vélo? Ou s'agit-il d'une opération de communication, destinée à promouvoir la carrière nationale d'un maire en quête de visibilité et à signaler une ville moyenne à l'heure d'un regain d'intérêt pour ces agglomérations à l'échelon national ?

Cet article défend l'hypothèse que l'expérience des "vélos municipaux" de La Rochelle permet de comprendre les transformations sociales, politiques et institutionnelles à l'œuvre dans les villes au cours des années 1970 . Elle révèle en particulier la dynamique d'affirmation des maires urbains, à travers la figure de Michel Crépeau. Dans la construction de son identité politique $^{4}$, ce maire s'appuie sur le vélo non pour le promouvoir en tant que tel mais pour certaines fonctions qu'il assume, notamment le déve-

2. Michel Crozier et Jean-Claude Thoenig, "La régulation des systèmes organisés complexes ", Revue française de sociologie, 1975, vol. 16, $\mathrm{n}^{\circ} 1$, p. 3-32.

3. Maxime Huré, Les Réseaux transnationaux du vélo. Gouverner les politiques du vélo en ville. De l'utopie associative à la gestion par les grands groupes privés (1965-2010), thèse de doctorat de science politique, sous la direction de Renaud Payre, Université Lyon 2, 2013.

4. La notion d'identité politique est ici mobilisée de manière large comme «une marque symbolique par laquelle l'acteur politique se distingue de ses pairs», Annie Collovald, "Identité(s) stratégique(s) ", Actes de la recherche en sciences sociales, vol. 1, $\mathrm{n}^{\circ} 73,1988$, p. 29-44. Cette identité est une construction constamment redéfinie et ouverte en fonction des interactions entre les acteurs politiques et des scènes où elle s'élabore. 
loppement économique et l'aménagement urbain. Ces nouvelles préoccupations municipales, dans des domaines encore largement investis par l'État dans les années 1970, interrogent également les relations entre échelles de décision. On y observe la diversification des acteurs et des formes de l'expertise, sans pour autant que les acteurs étatiques délaissent totalement un objet qu'ils perçoivent comme une expérimentation.

\section{Les origines des vélos municipaux de La Rochelle: entre opportunités politiques et critiques urbaines}

L'expérience rochelaise naît de la convergence entre plusieurs dynamiques. D'une part, le croisement entre l'ambition locale d'un jeune maire et l'intérêt renouvelé de l'État pour les villes moyennes; d'autre part, l'évolution du contexte socio-politique après Mai 1968, dans lequel se mêlent à la fois une expression écologiste nouvelle et les pratiques d'une société de consommation de masse.

UNE INNOVATION URBAINE AU CROISEMENT ENTRE LA MUNICIPALITÉ ET L'ÉTAT

Au cours des années 1970, La Rochelle entend se positionner comme une ville pionnière dans les domaines touchant à la notion alors émergente de "cadre de vie » ou relevant du ministère de la Protection de la Nature et de l'Environnement, créé en 1971. L'année précédente, elle s'est dotée d'un secteur sauvegardé en centre-ville5. En 1975, elle est la première ville française à établir un véritable secteur piétonnier autour de la rue du Temple, prolongeant ainsi l'innovation rouennaise de la rue du GrosHorloge, datant de 1971. D'autres thèmes font également l'objet d'une politique municipale, comme le tri du papier ou l'installation du chauffage solaire. Ces différentes opérations ont un moteur double: il s'agit de fortifier le civisme des Rochelais et, simultanément, de conférer à La Rochelle l'image d'une ville qui sait "devancer l'événement» ${ }^{6}$.

Cette politique environnementale est notamment liée à la personnalité de Michel Crépeau, maire radical de gauche, élu en 1971, à 40 ans. Il choisit de faire du territoire de sa commune le reflet de sa capacité à

5. Arrêté du 14 janvier 1970 portant création et délimitation d'un secteur sauvegardé à La Rochelle (Charente-Maritime).

6. "Les expériences rochelaises", La Rochelle Info. Bulletin municipal d'information rochelaise, $\mathrm{n}^{\circ}$ 6, décembre 1977. Voir aussi Josette Barbieri, "La leçon de La Rochelle», L'Unité, $\mathrm{n}^{\circ}{ }_{126}$, 4-10 octobre 1974, p. 21-22. 
anticiper les questions d'avenir. Il se bâtit ainsi une réputation qui lui permet d'appuyer sur la sensibilité écologiste naissante son positionnement politique à la marge des poids lourds que sont les partis socialiste et communiste français, tout en ancrant à gauche son radicalisme. Il sait par ailleurs employer la presse pour alimenter cette identité politique et devient progressivement l'un des habitués de ce jeu médiatique. Il travaille également son ancrage local, marqué par son inscription comme avocat au barreau de La Rochelle de 1955 à 1973.

L'action du maire est largement soutenue par un État qui engage alors une nouvelle politique urbaine touchant plusieurs secteurs, comme l'habitat insalubre, tout en poursuivant certaines dynamiques antérieures, comme celle des villes nouvelles ${ }^{7}$. À la suite de la circulaire signée par Olivier Guichard le 30 novembre 1971 protégeant les villes moyennes des "tours [et] barres " ${ }^{8}$, l'État amorce une politique en leur faveur, inscrite dans "l'urbanisme de plan ${ }^{9}$ qui prévaut alors. Pour la porter, un groupe interministériel des villes moyennes est créé le 4 juillet 1973. L'idée générale de cette politique est de soutenir ce maillon spécifique, vu comme capable d'équilibrer le territoire et d'éviter ainsi une concentration toujours plus forte dans les grandes agglomérations.

Cette politique choisit de concentrer les énergies sur les centres-villes, dont l'amélioration qualitative doit permettre d'accroître l'attractivité des villes moyennes. Elle se traduit donc par la mise en place de dispositifs liés à la mobilité, comme la piétonnisation ou la création de parkings, mais aussi par une rénovation du cadre bâti et une attention portée au dynamisme commercial de ces zones. Le plan se traduit par des subventions accordées à des projets négociés avec les villes, l'État proposant également d'apporter l'expertise technique qui pouvait manquer aux villes de cette taille. C'est dans ce cadre que s'inscrit le lancement d'une opération de mise à disposition de vélos dans le centre-ville de La Rochelle, portée par son maire mais largement financée par des fonds nationaux, reflet d'une « régulation croisée ${ }^{10}$ des relations entre collectivités et État.

7. Sur ce tournant, voir notamment «La résorption de l'habitat insalubre: retour sur une politique publique des années 1970. Actes du colloque du 29 juin 2012 », Pour Mémoire, horssérie, janvier 2013.

8. Circulaire du 30 novembre 1971 relative aux formes d'urbanisation adaptées aux villes moyennes.

9. Jean-Pierre Gaudin, «La genèse de l'urbanisme de plan et la question de la modernisation politique», Revue française de science politique, vol. $39, \mathrm{n}^{\circ} 3,1989$, p. 296-312.

10. Michel Crozier et Jean-Claude Thoenig, "La régulation...», op. cit., p. 3-32. 
Écologie Et BANALisation: De NOUVElles aspirations SOCIALES À L'ÉCHELLE MUNICIPALE?

Dans un contexte marqué par l'intérêt porté au vélo en ville par les premiers mouvements écologistes ${ }^{11}$, quelles sont les origines du projet? Un article du Monde signale deux expériences précédentes desquelles La Rochelle est réputée s'inspirer ${ }^{12}$. La première est celle des Provos, un mouvement libertaire et écologiste néerlandais qui, après l'avoir proposé sans succès en 1966 à la municipalité d'Amsterdam, instaura lui-même un système de vélos peints en blanc mis librement à disposition des habitants $^{13}$. La seconde est plus institutionnelle, puisqu'il s'agit des mini-vélos proposés en location gratuite aux personnes travaillant au marché de Rungis en 1970 - expérience qui dut toutefois cesser en raison des disparitions et des dégradations.

À l'échelle rochelaise, la location gratuite de vélos - c'est ce qui est alors envisagé - se comprend comme la troisième étape d'une politique générale de réaménagement des circulations. La première est la piétonnisation d'un réseau de rues centrales, rapidement suivie de la mise en place d'un nouveau plan de circulation automobile. Mais, du fait de l'étroitesse particulière des rues du centre-ville, le problème le plus aigu est bien celui du stationnement plus que celui de la circulation. Il convient donc de proposer un dispositif permettant aux automobilistes de laisser leur véhicule dans l'un des parcs de stationnement de la périphérie, jusque-là peu utilisés. Les vélos sont donc d'abord conçus comme un nouveau maillon de la chaîne des mobilités, dont la spécificité est de relever d'une idéologie politique naissante, l'écologie, et d'une philosophie novatrice.

Le projet repose sur la notion de «banalisation ", un terme régulièrement employé par ses promoteurs pour décrire une opération également dénommée "vélos pour tous ». La "banalisation" signifie la mise à disposition pour tous, partout et à tout moment, de vélos gratuits. Deux influences sont ici décelables. D’une part, cette notion est héritée de l'idéologie écologiste et libertaire, comme celle adoptée par les Provos à Amsterdam. Le vélo, icône d'une société alternative décrite par Ivan Illich ${ }^{14}$, incarne ces idéaux dans le champ de la mobilité urbaine. Son utilisation collective prévue par l'opération municipale permet même de dépasser la

11. Philippe Gaboriau, "Les trois âges du vélo en France», Vingtième siècle. Revue d'histoire, $\mathrm{n}^{0} 29,1991$, p. 17-33.

12. Marc Ambroise-Rendu, «Le vélo, ça vaut tous les centres aérés », Le Monde, 22-23 août 1976.

13. Yves Frémion, «Les Provos, 1965-1967», Matériaux pour l'histoire de notre temps, vol. 11, $\mathrm{n}^{\circ} 1,1988$, p. 48-52.

14. Ivan Illich, La Convivialité, Paris, Le Seuil, 1973 et Ivan Illich, Énergie et équité, Paris, Le Seuil, 1975. 
propriété individuelle qui est généralement d'usage. En outre, Mai 1968 favorise l'émergence du vélo sur la scène publique. Des associations environnementales comme Les Amis de la Terre manifeste à Paris en 1971, rassemblant plus de 10000 cyclistes ${ }^{15}$, puis des associations d'usagers du vélo se développent à partir de 1974 à Paris, Strasbourg ou Grenoble. Leurs revendications portent souvent sur une nouvelle conception de l'espace public et de l'aménagement urbain contre la séparation systématique des flux de circulation. Ces revendications trouvent un écho dans la sphère politique avec la candidature de René Dumont aux élections présidentielles de 1974, avant celles de nombreuses listes écologistes aux élections municipales de 1977. La bicyclette et ce qu'elle incarne s'inscrivent donc dans les nombreuses références idéologiques des mouvements écologistes français ${ }^{16}$.

D'autre part, la "banalisation" puise également ses racines dans un monde souvent décrié par le premier, celui du supermarché. De façon explicite, l'idée de libre-service s'inspire du caddie, cet objet devenu une icône de la société de consommation ${ }^{17}$. Sa facilité d'usage, le fait qu'il puisse passer d'un usager à l'autre selon les besoins, voire sa complémentarité avec la voiture, en font une source d'inspiration.

$\mathrm{Au}$-delà de ces origines contradictoires, le fonctionnement du système est placé dès ses débuts sous le signe de l'ambivalence. Le projet présenté en 1975 par le conseiller municipal, André Dubosc, prévoit un système de location, régulé par des agents municipaux qui pourraient confier des vélos à des utilisateurs en l'échange d'un document d'identité ${ }^{18}$. Ce système permettrait une tarification payante au-delà d'une première période gratuite. Cela ouvre également la porte à une tarification pour des usages de longue durée en dehors du centre-ville, notamment pour les touristes. Le fonctionnement libre, au contraire ne peut rapporter d'argent à la Commune et impose de restreindre les usages à un périmètre, si l'on ne veut pas les voir s'éparpiller bien au-delà de La Rochelle. Il permet toutefois une grande souplesse, si l'ensemble des utilisateurs joue le jeu. Le Conseil municipal discute de ces choix et se prononce début 1976 pour le fonctionnement libre.

Volontairement étroit, le périmètre correspond à la zone centrale où se posent les problèmes de congestion automobile ${ }^{19}$. Ses limites sont peintes sur la chaussée, afin d'être visibles par les cyclistes. Au sein de ce périmètre, le fonctionnement recommandé est celui du libre-service fondé sur

15. Jacques Durand, Vive le vélo, Paris, Éditions Stock, 1974.

16. Bruno Villalba, «La genèse inachevée des Verts", Vingtième siècle. Revue d'histoire, $\mathrm{n}^{\circ} 53$, 1997, p. 85-97.

17. Voir, par exemple, «Une idée qui a fait son chemin», Sud-Ouest, 9 août 1976.

18. AMLR, TEMP/9, "Vélos municipaux», 23 juin 1975.

19. Voir un plan dans «Les vélos pour tous, oui! Mais comment?», Sud-Ouest, 12 août 1976. 
un réseau de 23 stations où les utilisateurs peuvent retirer librement un vélo et doivent y déposer celui dont ils n'ont plus l'usage. Toute tentative de privatisation, plus ou moins intentionnelle, est interdite: stockage dans un lieu privé, pose d'un antivol, etc. De ce point de vue, le système se veut donc libertaire au sens où il combat toute appropriation. Mais il requiert simultanément un fonctionnement par stations, qui contraint les utilisateurs, contraintes rapidement non respectées. Les vélos, peints en jaune ${ }^{20}$, ne doivent pas non plus être utilisés en dehors des créneaux horaires établis, notamment le soir, lorsque les vélos sont parqués par les agents municipaux. Cette suspension du service le soir ne traduit-elle pas une peur de pratiques urbaines nocturnes incontrôlées? Le système inauguré le 14 août 1976 dans une ambiance festive, où se croisent les différents acteurs impliqués dans le projet, n'est donc pas sans ambiguïtés.

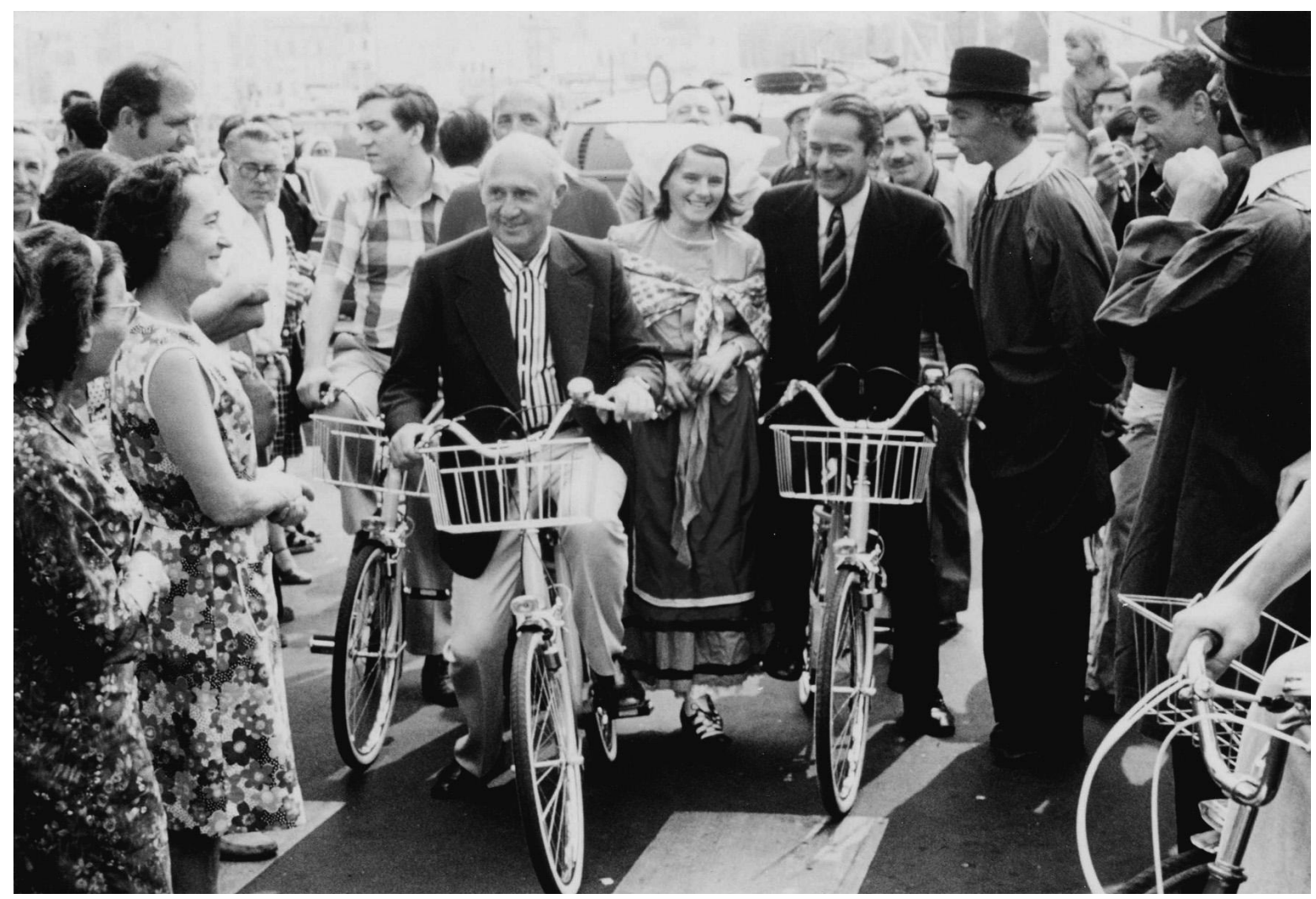

Figure 1 : Lucien Neuwirth (à gauche) et Michel Crépeau (à droite) lors de l'inauguration le 14 août $1976^{21}$.

20. Les 250 vélos jaunes sont de marque Gitane, une firme de Loire-Atlantique, présentée comme locale. Cette livrée est à l'origine de leur appellation courante; voir "À l'Arsenal: la veillée d'armes », Sud-Ouest, 10 août 1976.

21. AMLR, 28 FI 4302. 


\section{La mise en cuvre des vélos municipaux: un changement dans l'action publique urbaine?}

Le maire, dont la figure est centrale dans le projet, doit progressivement prendre en compte l'émergence de nouveaux acteurs, une fois les vélos mis en service, notamment les usagers. Si Michel Crépeau incarne l'opération sur le champ médiatique et politique national, à l'échelle locale, les « vélos municipaux" sont le support d'usages complexes, qui témoignent bien souvent d'un débordement du cadre défini initialement. Une double évolution de l'action publique urbaine est ainsi décelable.

CoNSTRUCTION POLITIQUE DE L'INNOVATION ET AFFiRMATION DU POUVOIR MUNICIPAL

L'opération s'inscrit dans un cofinancement entre l'État, puisque le secrétariat d'État aux Transports tenu par Marcel Cavaillé prend en charge $60 \%$ des coûts, et la Ville de La Rochelle, qui emprunte les $40 \%$ restants $^{22}$. L'investissement est de 100 ooo F (62 380 € 2014); il comprend une étude globale de stationnement, signe du lien structurel entre l'opération des "vélos municipaux", initialement appelée "autos-vélos", et la question automobile. Cependant, le dispositif s'identifie assez largement à la seule figure de Michel Crépeau, déjà atypique par sa volonté d'innovation ancrée dans un territoire local. C'est lui qui enfourche un vélo pour ouvrir la voie aux utilisateurs à venir mais aussi illustrer le caractère innovant d'une ville presque utopique:

«Ce genre d'initiative [...] se place dans le cadre de la qualité de la vie. Nos villes ont souffert trop longtemps de la suprématie de l'automobile. Il nous faut aujourd'hui découvrir des modes de transports mieux adaptés à nos rues. Libérer les hommes de leurs contraintes, les rendre heureux dans la liberté et la dignité, tel est le but que nous poursuivons. ${ }^{23}$

L'objectif médiatique est primordial, à tel point qu'il est revendiqué comme l'un des succès des "vélos municipaux" ${ }^{24}$. La presse locale offre un très large écho à l'opération, en particulier Sud-Ouest. Ce quotidien est alors engagé dans le développement de son implantation locale notamment en Charente-Maritime, après l'absorption de son ancien rival $L a$ France - La Nouvelle République ${ }^{25}$. Si sa ligne politique générale n'est

22. Conseil municipal de La Rochelle, séance du 4 novembre 1975.

23. "La balade des cyclistes heureux", Sud-Ouest, 16 août 1976.

24. "Michel Crépeau: une opération éducative», Sud-Ouest, 6 avril 1977.

25. Louis-Guy Gayan, «Sud-Ouest: du journal au groupe», Communication et langages, $\mathrm{n}^{\circ} 82$, $4^{\mathrm{e}}$ trimestre 1989, p. 79-92. 
habituellement pas tranchée, il prend clairement position en faveur de cette initiative du maire de La Rochelle ${ }^{26}$.

L'opération attire aussi la presse nationale, notamment Le Monde, sous la plume de Marc Ambroise-Rendu, lui-même impliqué dans le mouvement environnementaliste ${ }^{27}$. L'aura de Michel Crépeau dépasse même le cadre hexagonal, puisque le New York Times s'attarde sur lui dès décembre 1976: "a French town is battling the auto with bicycles" affirme ainsi l'article, dont l'auteur a été reçu par le maire ${ }^{28}$. L'hebdomadaire américain Newsweek lui consacre également un article en juin 1977, au lendemain de sa réélection, qui dresse un portrait plutôt élogieux de cet "agitatormayor ${ }^{29}$, capable de créer un consensus autour d'innovations multiples.

À l'automne 1976, au reproche formulé par un Rochelais dénonçant une "politique de prestige », Michel Crépeau choisit de répondre en assumant son volontarisme: " [s]i vous préférez un maire incolore, gérant médiocrement une ville de province triste et endormie, allez habiter ailleurs, et en tout cas, ne votez pas pour moi. ${ }^{3 \circ}$ Oubliant de préciser que l'opération est rendue possible par le financement d'un État assez effacé, que seul le secrétaire général de la Préfecture représente à l'inauguration.

L'opération vise d'ailleurs à passer outre les clivages politiques, puisque le maire radical n'est pas le seul à chevaucher à cette occasion l'un des premiers vélos. Il est accompagné de Lucien Neuwirth, député UDR de la Loire et cofondateur, avec Michel Crépeau en 1975, du Comité national des usagers des cycles et cyclomoteurs (CNUCC) ${ }^{31}$. Cette association regroupe des intérêts divers du deux-roues motorisé ou non, de sa fabrication - notamment dans l'agglomération de Saint-Étienne siège de Manufrance - à son utilisation. Sa visibilité, assurée lors de l'inauguration, cherche justement à soutenir l'image du vélo à l'échelle nationale. Les archives consultées ne reflètent pas de rôle précis de l'association dans le montage de l'opération elle-même.

L'expérience des "vélos municipaux" a ainsi contribué à consolider l'identité politique de Michel Crépeau, identité qu'il façonne de concert avec celle de sa ville, comme le rappelle Le Monde en 1981: "M. Crépeau a sans doute agi pour La Rochelle, mais sa ville le lui a bien rendu en lui assurant les bases de sa notoriété. ${ }^{32} \mathrm{Au}$ cours de sa carrière, Michel

26. Voir, par exemple, «Auto butée contre vélo fûté !», Sud-Ouest, 12 août 1976.

27. Marc Ambroise-Rendu, "Le vélo...», op. cit.

28. James Francis Clarity, "A French Town Is Battling the Auto With Bicycles», The New York Times, 27 décembre 1976.

29. Carter Wiseman, "The Agitator-Mayor», Newsweek, 6 juin 1977, p. 53-54.

30. "Si vous préférez un maire incolore», Sud-Ouest, 25 octobre 1976.

31. Voir Marc Ambroise-Rendu, «La révolte des deux-roues», Le Monde, 24 février 1976.

32. Laurent Zecchini, «M. Michel Crépeau: faire la preuve», Le Monde, 24-25 mai 1981. 
Crépeau fit un usage important de cette identité politique, notamment en 1981 lorsqu'il devient ministre de l'Environnement du gouvernement Mauroy. Le Monde rapporte alors que, «de par l'originalité de sa gestion de maire de La Rochelle, [il] jouit d'une image de marque favorable dans les milieux et dans l'électorat écologistes", présentant les "vélos municipaux" comme l'une des bases de son statut de "premier maire écologiste de France ${ }^{33}$. On assiste ainsi à la construction d'une forme de marketing politique et territorial visant à assurer la notoriété conjointe d'un maire et de sa ville à travers des dispositifs innovants d'action publique. Il s'agit également pour le maire de capter un électorat écologiste émergent à la veille des élections municipales de 1977.

\section{L'APPROPRIATION PAR LES USAGERS: VERS UN CHANGEMENT D'APPROCHE DANS LA MOBILITÉ URBAINE?}

Les «vélos municipaux" connaissent un vif succès dès leur inauguration. Leur appropriation par les usagers nous renseigne sur l'émergence plus générale de ces acteurs dans la construction des politiques publiques municipales au cours des années $1970^{34}$. Leur prise en compte dans les politiques de déplacements urbains trouve son origine dans les mouvements de défense du cadre de vie ${ }^{35}$.

Les pratiques viennent immédiatement souligner les biais d'une opération conçue comme un moyen de dégager le centre-ville du trafic automobile et non comme promotion du vélo en soi. Par exemple, l'étroit périmètre n'englobe pas un pôle aussi important que la gare, il est vrai légèrement excentrée à La Rochelle. Les utilisateurs ont vite fait de dépasser les limites imposées initialement aux vélos et ce, dès l'inauguration. La municipalité a décidé de ne pas sanctionner ces abus, mais de faire relever les noms des utilisateurs circulant en dehors du périmètre, qui reçoivent un courrier leur rappelant les règles d'usage et les sanctions encourues $^{36}$. Les Rochelais prennent aussi l'habitude de déposer leur vélo en bas de chez eux plutôt que dans l'une des stations, sans pour

33. Laurent Zecchini, «Le P.S. adopte une position de fermeté à l'égard des radicaux de gauche ", Le Monde, 22 mai 1981.

34. Éric Le Breton, Les Transports urbains et l'utilisateur: voyageur, client ou citadin?, Paris, L'Harmattan, 2002.

35. Mathieu Flonneau, «Parisiens, citadins, citoyens et automobilisme: du rôle de quelques associations dans la ville", dans Claire Andrieu, Gilles Le Beguec et Danielle Tartakowsky (sous la direction de), Associations et champ politique, Paris, Publications de la Sorbonne, 2001, p. 611-624.

36. AMLR, TEMP/9, Commission extra-municipale, «Ville de La Rochelle. Opération "Vélos municipaux" ", 10 janvier 1977. 
autant être sûrs de pouvoir le retrouver. Ainsi les pratiques font-elles émerger la contradiction entre la volonté de maîtriser territorialement les vélos et l'esprit d'un système en libre-service.

L'idée de "banalisation" accentue le phénomène classique de redéfinition d'un dispositif par ses utilisateurs au-delà des anticipations des décideurs, selon un processus de traduction ${ }^{37}$. Le système semble échapper à la maîtrise de ses concepteurs, jusqu'à faire figure d'objet autonome, capable d'influencer le débat et les représentations. Les contraintes spatiales et temporelles supposées le cadrer sont rapidement dépassées, si bien qu'il finit par vivre son existence en dehors de tout contrôle. Par exemple, tous les soirs, environ 120 vélos échappent au parcage nocturne ${ }^{38}$. Comme l'écrit un rapport de la commission extra-municipale à propos de 48 vélos recensés comme absents: "nous ne pouvons les considérer comme réellement disparus, pensant qu'ils sont utilisés à des fins personnelles et qu'un certain nombre tout au moins seront remis en circulation. » 39

L'appropriation sociale du dispositif est difficile à gérer pour la municipalité, de sorte qu'elle s'appuie sur une commission extra-municipale pour réguler les relations avec les usagers. On assiste à une forme de gestion du pluralisme qui permet aux élus de garder la main sur l'organisation des dispositifs en faveur du vélo tout en ouvrant la décision aux usagers. Cette gestion a deux conséquences. La première est que les commissions légitiment l'action des élus et des représentants des usagers dans l'action publique municipale. La deuxième est une transformation de certains dispositifs et de la manière de produire l'action publique avec la participation de différents acteurs.

\section{De la «banalisation» aux multiples formes de privatisation du système}

La gestion de l'opération innove effectivement par le recours à une commission extra-municipale, reflet de la multiplicité des acteurs impliqués dans sa régulation. Mais, à long terme, c'est par l'évolution du système lui-même que les dynamiques privées se manifestent, notamment avec la mise en place des vélos bleus.

37. Sur la sociologie de la traduction, voir notamment Michel Callon, "Éléments pour une sociologie de la traduction: la domestication des coquilles Saint-Jacques et des marins pêcheurs dans la baie de Saint-Brieuc ", L’Année sociologique, $\mathrm{n}^{\circ}$ 36, 1986, p.169-208.

38. "Opération "Vélos municipaux". Me Crépeau annonce de nouvelles mesures", Sud-Ouest, 26 août 1976.

39. AMLR, TEMP/9, Commission extra-municipale, «Ville de La Rochelle. Opération "Vélos municipaux" ", 10 janvier 1977. 


\section{UNE COMMISSION EXTRA-MUNICIPALE POUR GOUVERNER LE PLURALISME}

Il semble que cette commission ait été constituée en amont de l'opération, probablement dès $1975^{4 \circ}$. Elle regroupe des intérêts divers: animée par deux élus dont André Dubosc, elle réunit des profils variés : un ingénieur de la voirie, le directeur du syndicat d'initiative, le président du comité d'animation et de défense des intérêts de Tasdon, un quartier en dehors du centre-ville, etc. ${ }^{41}$. Les archives consultées ne permettent pas d'établir pourquoi Michel Crépeau a fait le choix de confier à une commission extra-municipale le pilotage du projet. S'agissait-il de contourner des réticences internes à l'équipe municipale ou aux services techniques? L'externalisation avait-elle pour objectif de mieux contrôler le projet? L'ouverture de la réflexion à un large cercle visait-elle à faciliter l'appropriation des vélos? Autant d'éléments qui ne sont pas nécessairement contradictoires entre eux. La commission extra-municipale offre simultanément un outil de gestion de l'opération, mais aussi et dès avant son lancement un lieu de débat autour du projet, où la controverse peut se déployer. Cette commission a un rôle de proposition auprès de l'exécutif municipal, qui fait savoir qu'il suit ses préconisations ${ }^{42}$.

L'utilisation collective d'un mode de déplacement individuel, la gratuité des bicyclettes et la surveillance du système par les usagers eux-mêmes ont engendré de nombreuses contestations, teintées de tensions idéologiques et de débats sur l'organisation des transports. Observé dès les débuts $\mathrm{du} \mathrm{Xx} \mathrm{x}^{\mathrm{e}}$ siècle, le gouvernement des mobilités urbaines par commission conduit les usagers à façonner techniquement les dispositifs en jeu ${ }^{43}$. L'opération des "vélos municipaux », conceptualisée et mise en œuvre par l'administration, déclenche des réactions de groupes d'acteurs qui s'organisent. En 1976, l'autorégulation sociale du système des "vélos municipaux» et la confiance du maire envers ses concitoyens, portée par les médias, provoquent ainsi une série de réactions. Gratuité et «banalisation" se trouvent discutées dans un cadre défini par la municipalité.

La commission extra-municipale permet, aux acteurs impliqués de participer à la formulation des problèmes et à la traduction des enjeux du

40. Les travaux de cette commission n'ont pas été conservés par les Archives municipales.

41. Elle est composée d'une quinzaine de membres, probablement des habitants, des représentants d'associations d'usagers et des commerçants; voir Les Vélos à La Rochelle. Conception d'une expérience et suivi de l'opération - juillet 1976 à juillet 1978, Bagneux, Cetur, 1978, p. 15.

42. "Prolongement et adaptation de l'expérience des vélos municipaux», La Rochelle Info. Bulletin municipal d'information rochelaise, $\mathrm{n}^{\circ} 6$, décembre 1977.

43. Voir notamment Sébastien Gardon, Gouverner la circulation urbaine: des villes françaises face à l'automobile (années 1910 - années 1960), thèse de doctorat de science politique, sous la direction de Gilles Pollet, Université Lyon 2, 2009, p. 265-345. 
dispositif. Ce processus leur confère un nouveau rôle dans l'élaboration des politiques publiques urbaines. Il légitime également l'action municipale dans la prise en charge de la question de la bicyclette. Les débats portent essentiellement sur trois points: l'idéologie collectiviste véhiculée par le dispositif, les coûts du système, bien supérieurs aux prévisions ${ }^{44}$, et l'évaluation de son efficacité pour les déplacements dans le centre-ville. Ces trois aspects reflètent aussi les différentes approches qui traversent la gestion des transports urbains dans les années 1970, à savoir un fort courant issu de la socio-économie ${ }^{45}$ et l'approche associative issue des différentes sensibilités écologistes.

Ces débats mobilisent les usagers, certaines critiques étant reprises par l'opposition municipale ${ }^{46}$. C'est surtout l'idéologie véhiculée qui heurte certains habitants. D'un côté "le libéralisme de l'opération" est dénoncé, de l'autre, on pointe "une dérive collectiviste», "les vols» ou une appropriation à "des fins politiques", si bien que la commission extra-municipale, «encouragée par des mouvements d'opinion [se prononce] pour l'application de sanctions $» 47$.

Dès le 26 août 1976, Michel Crépeau annonce les premiers ajustements : clarification du périmètre par l'installation de panneaux verticaux complétant les indications au sol et choix à terme de vélos plus solides. L'enjeu majeur pour l'avenir du dispositif touche à l'étroitesse du périmètre. Plutôt que d'entériner une situation de fait - nombre d'utilisateurs naviguent dans La Rochelle sans se préoccuper du périmètre -, la commission extra-municipale reste attachée à l'idée qu'une extension suppose un changement du système, notamment l'augmentation du nombre de vélos, ce qui implique des coûts supplémentaires. Les faits semblent pourtant mettre en doute cette idée. C'est cependant dans cette optique que travaille la commission qui envisage dès le début 1977 que des commerçants achètent des vélos et $\mathrm{y}$ apposent leur publicité.

Que ce soit en raison de la saison - les cyclistes étant présumés ne pas pédaler l'hiver -, de la nécessité de réparer le parc ou des élections municipales de mars, le service des "vélos municipaux» est suspendu du 15 janvier au 6 avril 1977. Les services municipaux en profitent pour

44. $391648 \mathrm{~F}$ (244 $309 € 2014)$ pour deux années de fonctionnement, contre une prévision de $160000 \mathrm{~F}$ (99 $808 € 2014$ ) qui incluait l'investissement et le fonctionnement (voir Les Vélos à La Rochelle..., op. cit., p. 45).

45. Symbolisée notamment par le Laboratoire d'économie des transports; voir Harold Mazoyer, Les Calculs de la puissance. Socio-histoire d'une science de gouvernement: l'économie des transports (1960-1982), thèse de doctorat de science politique, sous la direction de Gilles Pollet, Université Lyon 2, 2011.

46. Conseil municipal de La Rochelle, séance du 3 juin 1976.

47. Les Vélos à La Rochelle..., op. cit., p. 28. 
effectuer un voyage aux Pays-Bas dont ils retirent notamment des idées sur le type de vélo adapté à un tel service.

Au printemps 1977, la réouverture s'accompagne d'un accroissement du parc disponible et d'une petite extension du périmètre à la gare, au tri postal et à la plage. Progressivement, l'idée qu'il ne s'agit pas tant de lutter contre la congestion automobile, mais de proposer un service de mobilité supplémentaire, éventuellement à usage de loisirs, fait son chemin. Michel Crépeau l'avoue alors clairement, de même qu'il indique sa ligne politique: "Si l'on n'a pas résolu le problème de la circulation dans le centre on a au moins réussi l'opération publicitaire. Le nom de La Rochelle a beaucoup voyagé avec ces vélos. On a surtout beaucoup œuvré dans un sens éducatif. " ${ }^{48} \mathrm{Il}$ s'agit bien de constituer une image de marque pour la ville à l'international, aussi bien qu'une identité politique pour son maire au niveau national. Mais suite à la victoire de Michel Crépeau aux élections municipales, les objectifs se réorientent d'un système en libre-service destiné prioritairement aux Rochelais vers un système plus contrôlé à visée également touristique.

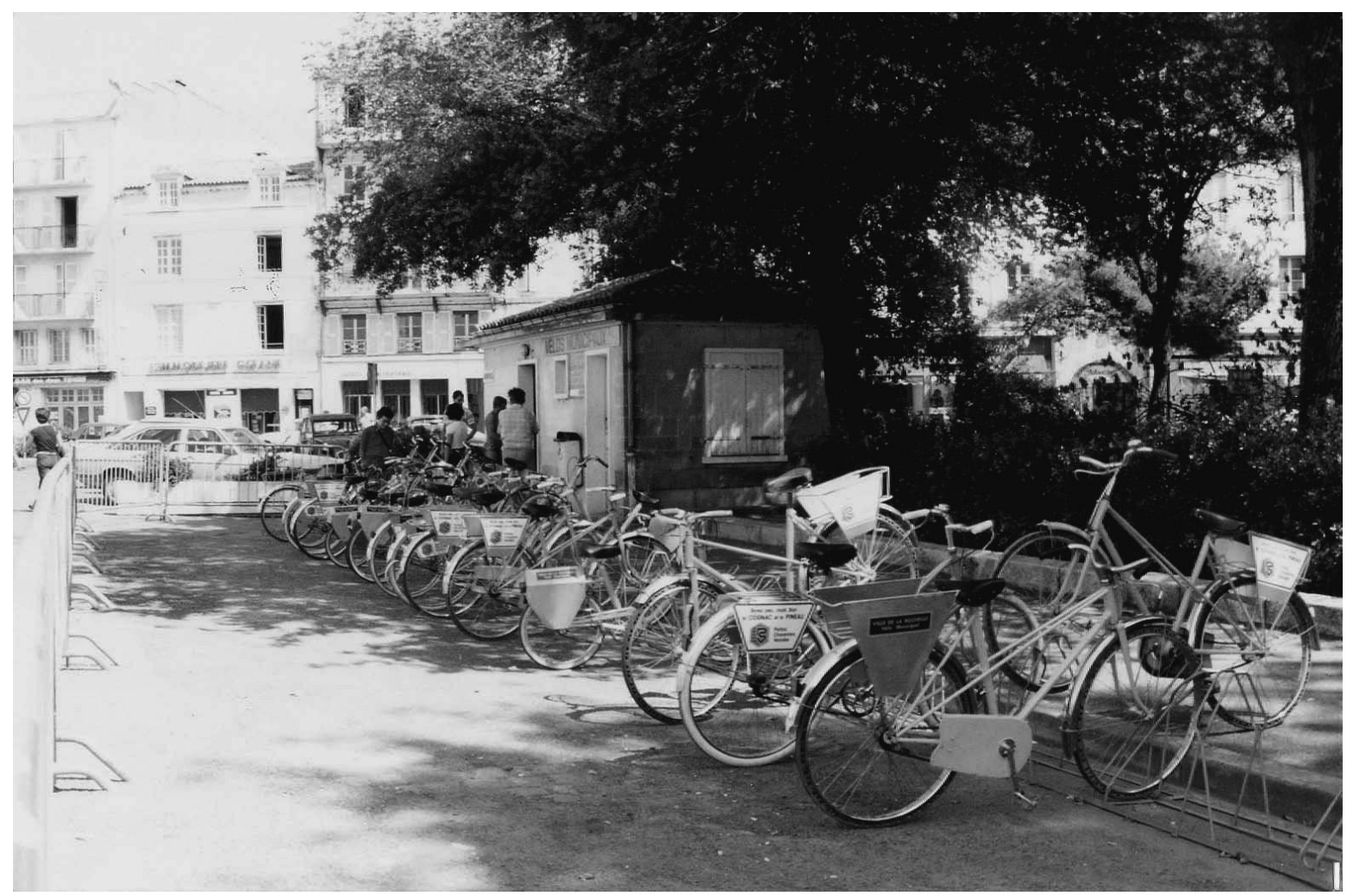

Figure 2: Une station de vélos municipaux en août $1981^{49}$.

48. "Michel Crépeau: une opération éducative», Sud-Ouest, 6 avril 1977. 49. AMLR, 28 FI 300. 
VÉlOS BLEUS ET PUBLICITÉ: LES INTÉRÊTS PRIVÉS

ET LA FIN DE LA «BANALISATION"

L'offre de mobilité de La Rochelle s'accroît le 6 août 1977 avec l'ouverture d'un service de vélos en location gratuite au nouveau port des Minimes, éloigné de 2 à $3 \mathrm{~km}$ du centre-ville. De même que leurs aînés jaunes, ces vélos bleus sont conçus comme un système d'intermodalité : on laisse son bateau au port, comme on laissait sa voiture en dehors du centre-ville, à ceci près que les vélos bleus sont réservés aux plaisanciers qui doivent mentionner un numéro de bateau pour pouvoir en bénéficier et ainsi rallier le centre alors qu'ils ne disposent pas de voiture. L'opération est conduite par la régie du port de plaisance, une jeune structure née en 1972.

Ces nouveaux venus, surnommés "vélos-régie», sont le signe d'une tentative de reprise en main de l'institution sur les vélos. Comme l'explique Sud-Ouest, "les vélos bleus ne sont pas de ces bicyclettes publiques, qu'on peut trouver [au] coin d'une rue, emporter et laisser à l'angle d'un quai. Ils sont numérotés, fichés, ils possèdent une identité gravée sur le cadre et peinte sur le carter ${ }^{50}$. Signe de cette évolution, les 25 exemplaires sont équipés d'un antivol.

On le voit, la municipalité amorce un virage dans sa politique avec l'opération des vélos bleus, sans oser toucher au principe de fonctionnement des vélos jaunes. Une initiative privée prend le relais pour ces derniers, avec l'ouverture d'un nouveau service de vélos en location gratuite dans certains parkings de la ville. Le Crédit agricole fournit 100 vélos équipés d'une publicité pour la banque, que les automobilistes peuvent louer dans des conditions qui accroissent encore le contrôle: après 3 heures d'utilisation, l'emprunteur doit payer 1 F (o,62 € 2014) par heure de retard. En outre, destiné à permettre aux automobilistes de faire leurs courses, le service a des horaires de fonctionnement restreints, de $9 \mathrm{~h}$ à $12 \mathrm{~h} 30$ et de $14 \mathrm{~h} 30$ à $19 \mathrm{~h}$; il est suspendu le dimanche et le lundi.

Ce nouveau dispositif entérine donc bien la fin de l'aventure libertaire ouverte par les vélos jaunes. Mais cette mutation n'est-elle pas également la garantie de la survie du système ? L'appui du privé permet de répondre à la question du financement du dispositif, de diffuser des vélos au-delà du périmètre initial et de dépasser ses ambivalences. Il n'est pas étonnant de voir émerger les enjeux commerciaux et publicitaires. En amont de l'opération des "vélos municipaux", les traces de l'implication de l'afficheur JCDecaux sont effectivement décelables dans les archives, même si elles 
demeurent ténues. Dès juin 1975, Jean-Claude Decaux, son patron, formule une offre pour la fourniture de 300 vélos par Manufrance, sans que sa lettre explicite le rôle qu'il espère tenir ${ }^{51}$. Le devis est obtenu par le biais de l'antenne lyonnaise de JCDecaux, dont le rôle dans l'essor de l'entreprise est essentiel ${ }^{52}$. Ayant développé une gamme d'abris d'autobus, devenus l'objet-clé de son offre commerciale, l'afficheur se lance dans une diversification de ses services et de ses mobiliers, qui intègre le vélo collectif. Il est d'ailleurs tout à fait remarquable de constater que JCDecaux est capable d'être présent en amont d'une opération aussi innovante, à moins qu'il n'en soit l'initiateur, hypothèse que les archives ne permettent pas d'étayer.

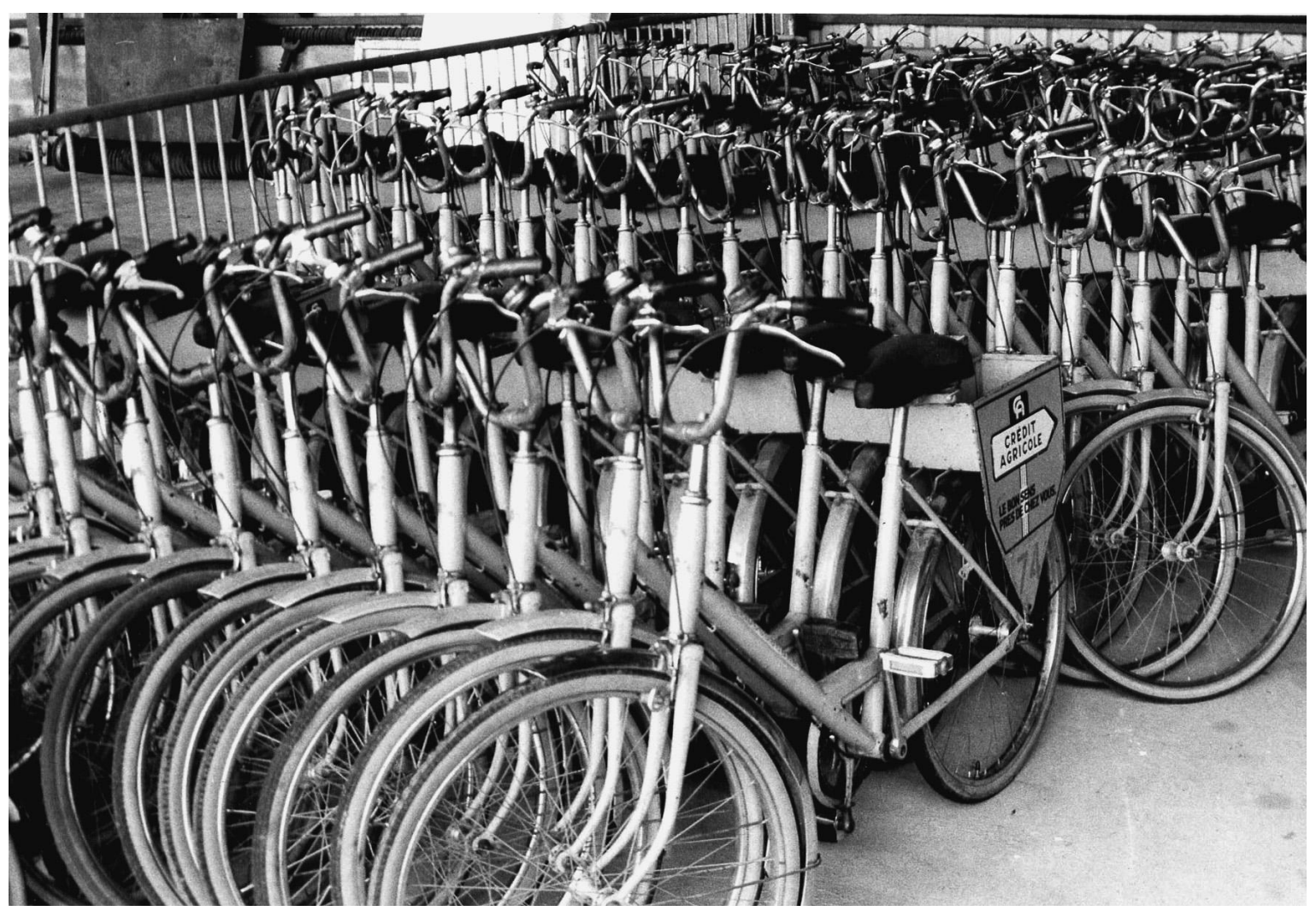

Figure $3:$ Le parc de vélos exploité en partenariat avec le Crédit agricole en janvier $1986^{53}$.

51. AMLR, TEMP/9, Lettre de Jean-Claude Decaux au maire de La Rochelle, 25 juin 1975. 52. Maxime Huré et Arnaud Passalacqua, «JCDecaux en sa "ville-fétiche". Mobilité et affichage à Lyon, de l'innovation à la dépendance (1965-2005)", dans Mathieu Flonneau, Léonard Laborie et Arnaud Passalacqua (sous la direction de), Les Transports de la démocratie, Rennes, Presses universitaires de Rennes, 2014.

53. AMLR, 28 FI 803. 
$\mathrm{Au}$-delà du rôle des acteurs privés dans les transformations du système rochelais, la mise en œuvre des "vélos municipaux" confronte deux visions de l'organisation des transports urbains. La première est portée par l'État, notamment par le ministère de l'Équipement, et consiste à répondre au problème de la sécurité des cyclistes. Elle s'appuie alors sur les plans de circulation en favorisant la séparation des flux de circulation. La seconde est conjointement soutenue par la municipalité de La Rochelle et les usagers. Elle s'appuie sur la «banalisation » pour faire la promotion du partage de l'espace public.

Dans ce contexte, l'État tente de reprendre la main à travers l'évaluation du dispositif, diffusée dès 1979 par Claude Gressier, alors directeur du Centre d'études des transports urbains (Cetur) au sein du ministère de l'Équipement ${ }^{54}$. Son montage est complexe, puisqu'il s'agit d'un travail fondé sur une commande confiée au CNUCC, dont la secrétaire générale, Véronique Granger, est aussi fonctionnaire du ministère ${ }^{55}$. L'étude recommande la fin de la «banalisation» et l'évolution du système.

Dans cette perspective, l'État n'est donc pas du tout absent de l'innovation en termes de mobilité urbaine. D'un côté, il autorise l'expérimentation de dispositifs à travers un soutien financier. De l'autre, il contrôle $a$ posteriori les résultats des expériences. Ce fonctionnement indique que les relations entre les municipalités et l'État ne découlent déjà plus du simple dirigisme étatique symbolisé par les plans nationaux en matière d'aménagement du territoire. On retrouve plutôt les prémisses de l'État régulateur, c'est-à-dire d'un État qui gouverne à travers des systèmes d'incitation et de contrôle auprès des collectivités ${ }^{56}$. Cependant, la réorientation des "vélos municipaux » après 1977 dans une visée touristique ne traduit-elle pas le souhait de la municipalité d'inscrire ce dispositif d'action dans un secteur d'activité davantage soutenu par l'État ${ }^{57}$ ?

Si l'expérience de La Rochelle peut être considérée comme révélatrice des changements dans les relations entre les municipalités et l'État, contribue-t-elle à transformer l'action même de l'État? Probablement pas, puisque l'État ne reconduit pas sa subvention au système rochelais et suspend même la quasi-totalité de ses financements à destination des

54. Les Vélos à La Rochelle..., op. cit.

55. Véronique Granger, La Rochelle. Expérience de banalisation de cycles, Paris, Ministère de l'Équipement, CNUCC, 1976, 45 p.

56. Pierre Lascoumes et Patrick Le Galès, Gouverner par les instruments, Paris, Presses de Science Po, 2004, p. 368.

57. Christophe Bouneau, «La promotion de l'économie touristique, levier de la politique d'aménagement du territoire en France des années 1950 aux années 1980 ", Entreprises et histoire, $\mathrm{n}^{\circ} 47$, 2007, p. 93-108. 
politiques du vélo en ville avec la décentralisation des années $1980^{58}$. Du côté de l'organisation des transports, le partage de l'espace public ne s'impose qu'au début des années 1980 au sein des services du ministère ${ }^{59}$. Pour sa part, le système rochelais est maintenu dans les années 1980, mais périclite progressivement jusqu'à l'inauguration du système Yelo en 2009, géré par le réseau des transports publics de l'agglomération. Si les "vélos municipaux" ont été précurseurs dans l'approche technique, politique et sociale des déplacements urbains, ils n'ont pas permis la diffusion de l'expérience dans l'ensemble des villes européennes.

Dans la France des années 1970, qui cherche de nouvelles voies pour ses politiques urbaines, La Rochelle se présente comme l'un des lieux de l'innovation. D'abord par cet objet singulier qu'est le vélo en libreservice, porteur de multiples ambivalences que résume cette notion de "banalisation". L'expérience a ainsi fait cohabiter une pluralité d'acteurs, aux intérêts et idéologies contradictoires, notamment les publicitaires et les usagers revendiquant une utilisation libre et banalisée. La souplesse de l'opération a donc assuré son succès, notamment pour les décideurs urbains. Ensuite, l'innovation - indéniable et multiforme - s'est heurtée à des freins, ce qui a conduit le système à se trouver progressivement en décalage avec les idéaux exprimés lors de sa conception, eux-mêmes déjà en partie dissonants.

Du point de vue des mobilités, l'expérience de La Rochelle a permis de formaliser un nouveau concept d'action, au croisement de différentes approches en termes d'aménagement urbain, de transport et d'expertise. S'il est malaisé d'établir la généalogie de ces expériences, après les "vélos municipaux", la publicité s'impose progressivement dans le financement des vélos en libre-service. C'est le cas à Copenhague où, à partir de 1989, circulent des vélos eux-mêmes supports de publicité ${ }^{60}$. Quant aux services proposés depuis une dizaine d'années par Clear Channel et JCDecaux, ceux-ci s'appuient sur le marché du mobilier urbain et de l'affichage publicitaire et dissocient physiquement le service de mobilité de son financement.

58. Maxime Huré, «La création d'un réseau de villes: circulations, pouvoirs et territoires. Le cas du Club des villes cyclables (1989-2009)", Métropoles, [En ligne], $\mathrm{n}^{\circ}$ 6, 2009, mis en ligne le 09 octobre 2009, URL: http://metropoles.revues.org/4010

59. Jean-Marc Offner, «L'expertise locale en transports urbains: entre logiques professionnelles et organisationnelles ", Politiques et management public, vol. 1, $\mathrm{n}^{\circ}$ 6, 1988, p. 81-102.

6o. Geneviève Laferrère, Les Politiques cyclables en Europe. Tour d'horizon des politiques nationales, Lyon, Certu, 2001. 
Ainsi, le vélo en libre-service rochelais doit davantage être compris comme une opération politique particulièrement réussie parmi les nombreuses innovations lancées alors par Michel Crépeau ${ }^{61}$. Dans la construction de son identité politique, le maire s'appuie sur le vélo pour s'assurer un électorat écologiste, renforcer l'image de sa ville et assurer sa promotion à l'échelle nationale et internationale. L'opération des "vélos municipaux " représente un instrument politique dont les usages se transforment suivant les contextes électoraux. D'abord vecteur de promotion de l'innovation sociale au niveau local et d'encadrement des controverses avant les élections municipales de 1977, elle permet l'intégration des acteurs économiques et le développement du tourisme aux échelles nationale et internationale après la réélection de Michel Crépeau. Le dispositif acquiert une visibilité nationale à l'approche des élections législatives de 1978, puis présidentielles de 1981. Il s'inscrit ainsi parmi les politiques urbaines menées par des municipalités de gauche, notamment à la suite de la «vague rose» de 1977, encore peu analysées mais dont le rôle dans la victoire de la gauche aux élections présidentielles et législatives de 1981 est perçu comme fondamental ${ }^{62}$.

Plus généralement, l'ascension politique de Michel Crépeau, étroitement liée aux "vélos municipaux", s'accompagne de transformations moins visibles dans l'action publique de la fin des années 1970. On assiste à l'affirmation d'un pouvoir municipal, s'appuyant sur des acteurs locaux, usagers et entreprises, pour apporter une expertise et des financements, autres que ceux proposés par l'État. Toutefois, l'État n'est pas évacué pour autant. D'abord par son rôle de financeur dans ce qu'il considère être une expérimentation sur laquelle il accroît son expertise d'évaluation de la mobilité urbaine. Mais aussi du fait que le niveau national demeure l'objectif d'un acteur local comme Michel Crépeau. Construire l'image de La Rochelle comme ville pionnière en se fondant sur des facteurs locaux participe d'une stratégie dont la visée finale est bien d'accéder à l'État. En ce sens, les valeurs des acteurs politiques auraient-elles évolué moins rapidement que les formes d'actions et les modalités de gouvernance?

61. Ont suivi le développement du covoiturage dans les années 1990, l'adoption de la voiture électrique par la municipalité en 1993 et la mise en œuvre de la première "journée sans voiture " en 1997.

62. Jean-Jacques Becker, Gilles Candar, Histoire des gauches en France, vol. 2, Paris, La Découverte, 2005 . 